\title{
TTR
}

Traduction, terminologie, re?daction

\section{L'interprétation et la traduction sur la côte ouest du Canada actuel à l'époque des explorations par mer et des débuts de la traite des fourrures sur le littoral (1774-1800) \\ Interpreting and Translation on the Canadian West Coast at the Time of the Maritime Explorations and the Early Days of the Fur Trade on the Coast, 1774-1800}

\section{Ginette Demers}

Volume 16, numéro 1, 1er semestre 2003

Controverse en traductologie

Controversy in Translation Studies

URI : https://id.erudit.org/iderudit/008563ar

DOI : https://doi.org/10.7202/008563ar

Aller au sommaire du numéro

Éditeur(s)

Association canadienne de traductologie

ISSN

0835-8443 (imprimé)

1708-2188 (numérique)

Découvrir la revue

Citer cet article

Demers, G. (2003). L'interprétation et la traduction sur la côte ouest du Canada actuel à l'époque des explorations par mer et des débuts de la traite des fourrures sur le littoral (1774-1800). TTR, 16(1), 215-241.

https://doi.org/10.7202/008563ar
Résumé de l'article

L'interprétation n'a pas joué un grand rôle au cours des explorations par voie maritime, les contacts avec les Amérindiens étant la plupart du temps très brefs. Le cas des marchands est différent. Comme la récolte de fourrures était plus abondante quand on pouvait communiquer avec les autochtones autrement que par signes, l'interprétation a vite pris de l'importance. En outre, presque tous les marchands ont laissé au moins un lexique bilingue. Au cours de la crise de Nootka, au début des années 1790, toutes les négociations territoriales menées par Vancouver, qui représentait la Grande-Bretagne, et Bodega, qui représentait l'Espagne, ont dû être traduites. L'interprétation et la traduction y ont donc joué un rôle essentiel.
Tous droits réservés @ C TTR: traduction, terminologie, rédaction — Les auteurs, 2003
Ce document est protegé par la loi sur le droit d'auteur. L'utilisation des services d'Érudit (y compris la reproduction) est assujettie à sa politique d'utilisation que vous pouvez consulter en ligne. 


\section{L'interprétation et la traduction sur la côte ouest du Canada actuel à l'époque des explorations par mer et des débuts de la traite des fourrures sur le littoral (1774-1800)}

\section{Ginette Demers}

Encore au début des années 1770 , le territoire qui forme aujourd'hui la Colombie-Britannique était connu des seuls Amérindiens qui l'habitaient. Pourtant, dès la fin du XVIII ${ }^{\mathrm{e}}$ siècle, le littoral était devenu familier à bien des Européens et des Américains. Entre 1774 et 1779, par exemple, les membres de trois expéditions espagnoles et d'une expédition britannique explorèrent la côte; à partir de 1785, un nombre sans cesse croissant de commerçants de fourrures appartenant à des nationalités diverses se mirent eux aussi à la fréquenter; au début des années 1790, enfin, la petite région de Nootka, située sur l'île de Vancouver, fut la cause d'un conflit entre la Grande-Bretagne et l'Espagne, et des représentants des deux pays y séjournèrent pour tenter de conclure une entente.

Les explorateurs, les marchands et les négociateurs eurent tous des contacts avec les Amérindiens. En outre, les pourparlers qui se déroulèrent à Nootka exigèrent la présence d'interprètes et de traducteurs. Dans le présent article, je décris les moyens qu'on utilisa pour apprendre les langues autochtones ou pour obtenir des interprètes traduisant vers ces langues. Je fais également état des activités d'interprétation et de traduction qui eurent lieu pendant la controverse de Nootka. Mon objectif est de cerner le rôle de l'interprétation et de la traduction au cours des explorations d'abord, puis dans les échanges commerciaux et les négociations territoriales. 


\section{Le contexte}

Au moment du premier contact avec des Européens, en 1774, au moins 80000 Amérindiens peuplaient la région comprise dans les limites actuelles de la Colombie-Britannique, et les deux tiers d'entre eux se trouvaient sur le littoral ou près de celui-ci. (Barman, 1996, p. 14) Comme le montre la carte qui figure en annexe, il y avait, du nord au sud, les Haidas et les Tsimshians, qui vivaient respectivement sur les îles de la Reine-Charlotte et sur le continent en face de ces îles; les Kwakiutls, qui occupaient l'extrémité nord de l'île de Vancouver, les îles voisines et, sur le continent, le centre du littoral; les Nootkas, établis sur la plus grande partie de la côte ouest de l'île de Vancouver; les Bella Coolas, installés près de l'embouchure des rivières Dean et Bella Coola; les Salishs de la côte, enfin, qui habitaient l'extrémité sud de l'île de Vancouver et la partie du continent lui faisant face. Ces nations ne présentaient aucune unité politique et parlaient des langues très différentes les unes des autres ${ }^{1}$, mais elles partageaient une culture caractérisée par l'importance du rang social et des richesses. Ainsi que le souligne Gibson, "Trade was well suited to Northwest Coast Society, for one of its main values and goals was the accumulation, display and redistribution of material goods ». (1992, p. 8)

Pendant la période observée, les explorateurs et les marchands côtoyèrent surtout les Nootkas, et plus particulièrement ceux qui vivaient sur les bords du détroit de Nootka. C'est aussi principalement à leur dialecte, le moachat, qu'ils s'intéressèrent. ${ }^{2}$

${ }^{1}$ Ces langues sont indiquées sur la carte en annexe. Notons que le haida, le tsimshian, le kwakiutl et le nootka pouvaient comporter des variations régionales importantes et que le pentlatch, un des dialectes parlés par les Salishs de la côte, est aujourd'hui disparu. (Duff, 1969, p. 15)

${ }^{2}$ Cela s'explique peut-être par le fait que Maquinna, le chef des Moachats, semble avoir été très accueillant. En outre, parmi les trois grands chefs qui se trouvaient sur l'île de Vancouver, il parait avoir été le plus puissant : « Because the leading chiefs and their closest associates were all related by marriage, $[\ldots]$ they could call upon the others to act as allies. Maquinna's membership in this network of alliances combined with his very high rank and social status meant that although he held no formal position of overall authority, in practice his influence could amount to that of an umbrella chief. » (Marshall, 1993, p. 163) 
En 1741, Vitus Bering avait découvert l'extrême nord de la côte ouest au cours d'un voyage d'exploration qu'il avait fait pour le compte de la Russie, et les Russes avaient par la suite construit des comptoirs de traite des fourrures dans les Aléoutiennes, îles qui font maintenant partie de l'Alaska. Leur présence en Amérique inquiétait les Espagnols, établis au Mexique et en Californie. C'est pourquoi, au début des années 1770, ceux-ci décidèrent d'organiser une expédition qui leur permettrait, s'ils constataient que leurs établissements pouvaient être menacés, de prendre possession du territoire s'étendant entre la mission de Monterey, en Californie, et le $60^{\mathrm{e}}$ degré de latitude, lequel se situe, sur le littoral, à quelque 600 kilomètres au nord de la frontière actuelle de la Colombie-Britannique.

Les Britanniques étaient eux aussi au courant des explorations et des activités commerciales des Russes. Ils n'ignoraient pas, non plus, les projets censément secrets des Espagnols. Il semble que cela ait ravivé chez eux «l'obsession du passage du nord-ouest», qu'ils cherchaient depuis plus de deux siècles : "By the mid eighteenth century the search had become so desperate that Parliament voted a reward of $£ 20,000$ - an enormous sum in those days - for discovery of a passage between Hudson Bay and the Pacific Ocean. » (Barman, 1996, p. 22) Cependant, au milieu des années 1770, personne n'avait encore pu réclamer le prix, et l'amirauté préparait une expédition qui aurait pour objectif de faire cette découverte.

À l'est des Rocheuses, enfin, les commerçants de fourrures s'approchaient de plus en plus des montagnes, mais celles-ci constituaient toujours une barrière : ce n'est qu'en 1793 qu'on réussirait à la franchir. Entre-temps, les États-Unis, qui avaient grand besoin d'argent à la suite de la guerre de l'Indépendance, s'intéressèrent vivement à la traite sur la côte nord-ouest. Ce pays finit même par dominer le commerce maritime des fourrures.

\section{Les premières explorations}

En 1774, un navire espagnol commandé par Juan Pérez partit de San Blas, sur la côte ouest du Mexique, et fit voile vers le nord pour explorer la côte. Au cours de ce voyage, personne ne put mettre pied à terre. Les marins firent un peu de traite avec des Haidas et des Nootkas qui s'étaient rendus près du navire dans leurs canots, mais si l'on se fonde sur les journaux tenus par les deux aumôniers qui accompagnaient Pérez, Fray Juan Crespi et Fray Tomás de la Peña, les 
Espagnols ne tentèrent pas de se faire comprendre autrement que par signes. $^{3}$

Comme Pérez avait dû rebrousser chemin avant d'atteindre le $60^{\mathrm{e}}$ parallèle, une deuxième expédition fut organisée l'année suivante. Deux navires, commandés respectivement par Bruno de Hezeta et Francisco de la Bodega y Quadra, quittèrent San Blas au printemps. Cette fois encore, les contacts avec les Amérindiens furent rares, d'autant plus que six marins qui étaient descendus à terre alors que les navires se trouvaient près d'une rivière qui porte aujourd'hui le nom de Quinault, dans l'État de Washington, furent attaqués et tués par des autochtones qui jusque-là s'étaient montrés amicaux. (Bodega, 1943, pp. 117-118) Hezeta fit demi-tour quelques jours plus tard. Bodega poursuivit l'exploration et longea la côte jusqu'au $58^{\mathrm{e}}$ parallèle. Il débarqua à quelques endroits pour prendre officiellement possession du territoire, obéissant ainsi aux instructions du vice-roi du Mexique, Antonio Maria Bucareli. (Ibid., p. 102) Il dut cependant se contenter de s'exprimer par gestes, même si ce procédé était loin d'être efficace : « [...] we had no intercourse but by signs, and therefore both parties often continued in a total ignorance of each other's meaning ", dit le pilote Francisco Antonio Mourelle. (1781, p. 20)

En mars 1778, le capitaine Cook, qui tentait de découvrir le passage du nord-ouest, jetait l'ancre dans le détroit de Nootka. Cook passa un mois à Nootka parce que ses navires avaient besoin d'être réparés, et cela lui permit d'observer le mode de vie des Amérindiens. Il avoue cependant ne pouvoir faire que des suppositions quant à leur forme de gouvernement ou à leur religion : « [...] we could gain nothing from information, as we had learnt little more of their language than to ask the name of things and the two simple words yes and no. » (1967, p. 322) Cook compila tout de même un lexique anglais-moachat de quelque 250 mots dans le but de voir si ce dialecte présentait des analogies avec ceux des groupes amérindiens qui vivaient à proximité. (Ibid., p. 323) Le petit vocabulaire, qui contient presque uniquement des mots concrets, peut paraître aujourd'hui très élémentaire. Néanmoins, à l'époque, il fut non seulement utile à de nombreux commerçants de fourrures, mais servit aussi de base à d'autres lexiques bilingues et fut même traduit en espagnol.

${ }^{3}$ Voir, par exemple, Peña, 1969, pp. 157, 161, 179 et Crespi, 1969, pp. 227, $229,239,255$. 
Les Espagnols avaient entendu parler de l'expédition de Cook dès 1777, mais c'est seulement en 1779 qu'ils purent effectuer un troisième voyage vers le nord. Ignacio de Arteaga longea la côte pendant deux mois afin d'en poursuivre l'exploration et de s'assurer qu'aucun navire britannique ne se trouvait dans les parages. Pour la première fois, les Espagnols essayèrent de communiquer avec des Amérindiens autrement que par signes. (Ces Autochtones appartenaient à la nation des Tlingits, qui habitent la côte de l'Alaska et, à l'intérieur des terres, le Yukon et le nord de la Colombie-Britannique.) Ils voulurent élaborer un lexique bilingue, mais n'y arrivèrent pas. Archer précise que d'après Mourelle, "the Spanish officers could not even write the names pronounced by the Natives or reproduce the answers to simple questions asked about objects $» !(1993$, p. 134) Arteaga décida donc d'acheter quelques enfants que les Amérindiens s'étaient montrés désireux de vendre ${ }^{4}$ dans l'espoir d'en faire des interprètes qui pourraient plus tard être utiles. Ce souhait, cependant, ne devait pas se réaliser : quatre des enfants moururent peu après, et le cinquième, qui avait été adopté par Bodega et emmené au Mexique, ne paraît pas être retourné parmi les siens. (Ibid., p. 135)

Il est intéressant d'ouvrir une parenthèse pour mentionner que les Espagnols achetèrent d'autres enfants entre 1789 et 1795, années pendant lesquelles ils occupèrent Nootka, pour des motifs humanitaires et religieux autant que pratiques. (Vancouver, 1984, p. 518, n. 1) Ils semblent être les seuls à avoir utilisé ce moyen d'obtenir des interprètes. En effet, si certains des commerçants de fourrures acquirent des esclaves ${ }^{5}$, rien n'indique dans la documentation que c'était pour qu'ils leur servent d'interprètes. Quant aux explorateurs britanniques, ils paraissent avoir trouvé ce genre de transaction répugnante. Vancouver, par exemple, dit à ce sujet: «This [...] I peremptorily prohibited, expressing, as well as I was able, our great abhorrence of such traffic. » (Ibid., p. 518)

\footnotetext{
${ }^{4}$ Il s'agissait sûrement d'esclaves. En effet, les peuples du littoral avaient une organisation sociale fortement hiérarchisée qui comprenait trois strates: les « nobles », les gens du commun et les esclaves. Ces derniers étaient acquis au cours de raids ou encore achetés. Selon Donald, en effet, le commerce des esclaves faisait partie intégrante de l'économie de ces Amérindiens. (1997, p. 141)

${ }^{5}$ Ainsi, le marchand John Meares note dans le récit d'un voyage effectué en 1786-1787 qu'il a acheté une jeune femme qui avait été capturée au cours d'un raid dans l'intention de la ramener dans sa tribu. (1967, pp. xxviii-xxix)
} 
Comme Arteaga n'avait trouvé aucune trace du passage des Britanniques, les Espagnols furent rassurés. Il allait s'écouler neuf ans avant que de nouvelles explorations soient effectuées.

\section{Les débuts de la traite des fourrures}

Les marins du capitaine Cook avaient acquis à Nootka des peaux de loutre de mer pour se tenir au chaud. À leur grande surprise, quand ils arrivèrent en Chine en 1779, celles-ci se vendirent avec un profit de $1800 \%$ ! La publication du journal de Cook, en 1784, fit connaitre ces profits énormes et provoqua une véritable ruée vers la fourrure : on estime qu'entre 1785 et 1800 , quelque 180 navires fréquentèrent la partie de la côte ouest qui s'étend au nord du $46^{\mathrm{e}}$ parallèle. (Cook, 1973, Annexe E)

Les marchands faisaient un commerce triangulaire : à partir de leur port d'attache, ils se rendaient sur la côte nord-ouest où ils troquaient des articles de traite contre des peaux de loutre de mer, puis ils se dirigeaient vers la Chine où ils échangeaient ces fourrures contre de la soie, du thé et des épices qu'ils vendaient ensuite dans leur pays. (Fisher, 1996, p. 51)

Les commerçants eurent recours à différents procédés pour arriver à communiquer avec les Amérindiens. Le plus courant fut la compilation de lexiques bilingues, lesquels pouvaient servir d'aidemémoire d'un voyage à l'autre ou encore, dans le cas des grandes sociétés de traite, être transmis d'un capitaine à l'autre. La valeur de ces travaux est évidemment inégale. Par exemple, celui que l'Américain Haswell élabora ne contient que 200 mots alors que le Britannique Strange ajouta plusieurs centaines de mots à celui de Cook. Le plus intéressant est peut-être le lexique moachat-anglais qui figure dans le récit de voyage d'Alexander Walker, un autre marchand britannique. On y trouve des noms ou expressions appartenant à plusieurs domaines différents - la culture matérielle et spirituelle des Nootkas, la botanique, la zoologie, l'anatomie, etc. - ainsi que de courtes phrases, une cinquantaine de verbes et les nombres jusqu'à 200. Les mots sont classés par thèmes et présentés sur trois colonnes : la première comprend les mots moachats transcrits par Walker, la deuxième les mots anglais correspondants et la troisième les équivalents fournis par Cook dans le cas des mots qui apparaissent 
également dans le vocabulaire compilé par ce dernier. L'extrait suivant illustre la configuration du lexique de Walker ${ }^{6}$ :

\begin{tabular}{|lll|}
\hline $\begin{array}{l}\text { Nootka as it } \\
\text { appeared to me }-\end{array}$ & - English - & $\begin{array}{c}\text { - According } \\
\text { to C. Cook }\end{array}$ \\
Enitsheetl & The moon & Onulszthl \\
Milkhleemootl & full moon & \\
Quelkhquatta & $\begin{array}{l}\text { the moon in } \\
\text { its increase }\end{array}$ \\
or decrease & \\
Tatooss & stars & \\
Nas or Naas & The skye $[$ sic $]$ & Nas \\
& & Eenaeehlnas \\
\hline
\end{tabular}

(1982, pp. 91-92)

Quelques remarques d'ordre grammatical accompagnent le lexique. Ainsi, Walker mentionne que les pronoms sont rares en moachat et que l'article y est complètement absent. (Ibid., p. 89)

Il arrive que l'équivalent proposé par ce marchand soit incorrect : rain, par exemple, correspond à O-okomahee, qui signifie beau temps! En outre, Walker a dû écrire les mots avec les seules lettres de l'alphabet romain, ce qui ne lui a pas permis de reproduire tous les sons du moachat. Il reste que, d'après les éditeurs du récit, ses transcriptions sont suffisamment justes pour qu'encore aujourd'hui on puisse reconnaître la plupart des mots. Ceux-ci estiment également que la découverte du lexique "nearly two hundred years after it was prepared will make a significant contribution to the understanding of the development of the Nootka Language ». (Ibid., pp. 250-251)

Le lexique de William Sturgis est également digne de mention, bien qu'il soit peu volumineux. En effet, c'est la seule liste trilingue qui ait été repérée, la seule également dans laquelle les données sont

\footnotetext{
${ }^{6}$ Les éditeurs mentionnent qu'ils ont respecté « the order and arrangement of the original ». (Walker, 1982, p. 278)
} 
présentées par ordre alphabétique. Les quelque 135 mots anglais qui y figurent sont suivis de leurs équivalents dans un dialecte haida et dans un dialecte tlingit. (Sturgis, 1978, pp. 108-112)

Le fait que la plupart des commerçants de fourrures ait laissé au moins un lexique bilingue laisse croire que ces travaux étaient considérés comme des outils précieux. Toutefois, la East India Company envisagea un moyen différent. On trouve en effet le passage suivant dans les instructions destinées à James Strange, qui dirigeait en 1786 l'expédition commerciale qu'elle avait organisée :

If You could in Your range along the coast of America land any of Your Crew who choose to turn out Volunteers on the occasion, in [some] centrical situation, with a promise of Returning to them again, we conceive it might be attended with singular advantage as by attaining the language thus, might in future prove very useful. We have therefore to desire that You will offer a proper pecuniary reward to any such Volunteers, and as a farther Inducement assure them of our rewarding them amply ourselves, as also of our recommending them in the manner such a Publick [sic] spirited Service merits. (Strange, 1982, p. 34)

Le marchand se rendit vite compte de la pertinence de cette suggestion, car il n'arrivait pas à faire comprendre aux habitants de Nootka le but véritable de son voyage :

[...] the Generality of them, conceived Our Visit proceeded from no other Cause, than a want of food [...] and they accordingly brought us no inconsiderable quantity of dried fish, which they offered for Sale, and which I as constantly rejected, as I wished to impress on their minds that my wants were Confined to the Article of Skins alone, and thereby make them more Zealous in their future exertions to procure as many of them as possible. (Ibid., p. 78)

Un jeune chirurgien, John Mackay, se porta volontaire et passa un an à Nootka, mais... ce n'est pas la East India Company qui récolta les fruits de ce séjour: Mackay s'embarqua sur le premier navire qui atteignit Nootka en 1787, lequel appartenait à Charles Barclay. Selon madame Barclay, qui accompagnait son mari, Mackay ne fut que trop heureux de se joindre à eux. La version de Mackay est différente : de retour en Angleterre, il dit à Strange que Barclay l'avait forcé à quitter Nootka. (Ibid., p. 22) Quoi qu'il en soit, grâce à leur interprète, les Barclay acquirent dans les environs immédiats de Nootka la plus grande partie des 700 peaux qu'ils vendirent en Chine. Bien des 
commerçants de fourrures eurent la même idée que la East India Company, mais les hommes qu'ils laissèrent sur la côte n'y restèrent jamais longtemps. En effet, d'après Howay, éditeur du journal du marchand James Colnett, "This was frequently tried but always failed $»^{7}$. (Colnett, 1940, p. 21, n. 2)

D'autres commerçants essayèrent d'avoir recours à des Amérindiens comme interprètes. Ainsi, en 1788, John Meares, le plus ambitieux peut-être des marchands britanniques, crut que le hasard lui avait fourni l'interprète idéal : il ramenait à Nootka Comekela, le propre frère du chef Maquinna, qu'il avait découvert en Chine. D'après Meares, qui ramenait aussi chez eux quelques habitants des îles Sandwich (Hawaii) et un autre Amérindien, " [they] had been brought to China, by different ships, rather as objects of curiosity, than from the better motive of instruction to them, or advantage to commerce $[. .]$.$» .$ (1967, pp. 9-10) Le marchand combla Comekela de cadeaux pour qu'il accepte de lui servir d'interprète et qu'il l'aide à obtenir une riche cargaison de fourrures à bas prix. Meares, toutefois, fut grandement déçu. Il dit en effet :

Comekela was, at first, very active in forwarding our commercial arrangement; but he had become very deficient in his native tongue, and he now spoke such a jargon of the Chinese, English and Nootkan languages, as to be by no means a ready interpreter between us and the natives; - - besides, in returning to the manners of his country, he began to prefer the interest of his countrymen, and [...] to forget the very great kindnesses we had bestowed upon him. (Ibid., p. 121)

La présence d'interprètes autochtones à bord des navires semble très rare à l'époque qui nous intéresse : le cas de Comekela est

\footnotetext{
${ }^{7}$ Les sources sont muettes sur les raisons de cet échec. Les marins étaient peutêtre incapables de s'adapter au mode de vie des Amérindiens, mais il est possible aussi que certains capitaines leur aient fait des offres qu'ils ne pouvaient refuser: au terme du long voyage, les équipages étaient parfois réduits parce que des marins avaient déserté pendant les escales et que d'autres étaient morts en mer. L'exemple suivant, bien qu'il s'agisse sans doute d'un cas extrême, montre que, dans ces circonstances, on était prêt à payer cher les services des marins. John Meares, dont la moitié de l'équipage était mort du scorbut au cours de l'hiver 1787, réussit à convaincre deux capitaines britanniques de lui céder un homme chacun. Ces hommes exigèrent quatre livres par mois au lieu des 30 shillings habituels, mais il fut forcé d'accepter. «I was at their mercy », dit-il. (Meares, 1967, p. xxv)
} 
le seul qui figure dans les sources consultées ${ }^{8}$. On aura plutôt recours à des marins qui, après avoir effectué quelques voyages sur la côte, avaient acquis une certaine connaissance des langues amérindiennes. L'anecdote suivante montre que ces marins étaient recherchés.

Les premiers navires américains, sous les ordres de Robert Gray et de John Kendrick, étaient apparus à Nootka cet été-là. Meares informa Gray, arrivé avant Kendrick, qu'il y avait eu une mutinerie à son bord et qu'il avait débarqué dix hommes, dont John Green, le meneur. Il lui fit promettre de ne pas le prendre avec lui, mais comme Green disait connaître les langues parlées sur la côte, la tentation était grande. Robert Haswell, maître d'équipage à bord du navire commandé par Gray, écrit en effet :

[...] such a man as this as we ware [sic] all ignorant both of coast and languages it naturally struck us might be servisable [sic] but Captain Gray had given captain Mears [sic] his word he would not take him on board while the snow was in the port. but [sic] we supplied him with provisions $[\ldots]$ and when the Columbia arrived he was taken as a seaman onboard of her. (Haswell, 1941, p. 57)

Green semble toutefois avoir été un fauteur de troubles; les Américains ne le gardèrent pas très longtemps. Ceux-ci, d'ailleurs, hivernèrent sur la côte, ce qui, selon Haswell, leur permit d'apprendre rapidement le moachat: "Our constant converse with the Natives enabled us to gain a considerable knowledge of their Language Manours and Customs I [...] have here insurted [sic] a vocabulary which enabled us to converse on allmost aney [sic] Subject with facility. ${ }^{9}$ (Ibid., p. 58) Ces paroles sont peut-être exagérées, mais

\footnotetext{
${ }^{8}$ Peut-être que les Amérindiens se méfiaient des marchands. C'est, en tout cas, ce que laisse supposer une remarque de Meares. Au cours de l'automne 1786, il demanda à un chef de permettre à un jeune homme de passer l'hiver à bord de son navire pour que lui et ses marins puissent apprendre la langue, mais le chef n'accorda pas l'autorisation : « [...] this proposition, however, they constantly refused; but on condition that we should leave one of our people with them. Indeed the King himself always hesitated to come on board, unless one of our seamen remained in the canoe during his visit. » (1967, p.xxviii)

${ }^{9}$ Le premier-maître, Joseph Ingraham, considérait lui aussi que ce séjour avait été profitable sur le plan de l'apprentissage de la langue : «[...] we remained among these people nine months and could converse with them perfectly well. » (1971, p. 220)
} 
plusieurs marchands allaient suivre l'exemple de Kendrick et de Gray au cours de la décennie suivante. À la fin du siècle encore, William Sturgis estimait qu'il était essentiel d'avoir une certaine connaissance des langues autochtones : " All intercourse with the natives was carried on in their own language, which compelled us to acquire a competent knowledge of it for managing trade, but not sufficient for a critical examination. » $(2000$, p. 38$)$

\section{La première occupation de Nootka par les Espagnols}

À l'insu des Britanniques et des Américains, deux navires espagnols s'étaient rendus jusqu'aux Aléoutiennes au cours de l'été 1788. Les Espagnols apprirent ainsi ce qui se passait à Nootka. En outre, le commandant de l'établissement russe de Kodiak, Eustrate Delarov, leur dit que les Russes avaient l'intention de s'y installer après en avoir chassé les Anglais et les Américains. (Cook, 1973, pp. 119-125) Le vice-roi du Mexique, Manuel Antonio de Flores, craignant d'être pris de vitesse, envoya une expédition dès le printemps 1789: le 5 mai, un navire de guerre commandé par Esteban José Martinez arrivait à Nootka. Martinez avait l'ordre de fonder un établissement temporaire en vue de faire reconnaître les revendications de l'Espagne sur la côte $\mathrm{du}$ Pacifique. (Le vice-roi désirait également qu'il ramène des Amérindiens au Mexique pour qu'on en fasse des interprètes, s'il trouvait des volontaires. (Cook, 1973, p. 122) Toutefois, s'il y en eut, ceux-ci ne semblent pas être revenus ${ }^{10}$.)

Pour s'assurer qu'aucun pays ne menaçait les droits supposés de l'Espagne, Martinez avait l'intention de faire traduire les instructions de tous les capitaines étrangers qui se présenteraient à Nootka. Kendrick réussit à le convaincre qu'il s'y trouvait pour radouber ses navires, et il ne fut pas inquiété. D'ailleurs, les relations entre les Américains et les Espagnols allaient être cordiales au cours des deux occupations de Nootka. Le fils aîné de Kendrick, John, se convertit même au catholicisme, changea son prénom en celui de Juan et servit d'interprète aux Espagnols pendant plusieurs années. Les Britanniques, toutefois, eurent des problèmes, et celui qui souffrit le plus de la situation est probablement John Meares. En effet, l'année

10 D'après les sources consultées, ce sont José Mariano Moziño et Juan Kendrick qui servirent d'interprètes vers le moachat de 1792 à 1795, année où les Espagnols quittèrent définitivement Nootka. Voir, par exemple, Moziño, 1970, pp. 55, 69 et Cook, 1973, p. 422. 
précédente, il avait obtenu du chef Maquinna l'autorisation de construire à Nootka une maison et un entrepôt. Il y avait également fait construire un petit navire. Martinez s'empara immédiatement de ces biens. De plus, si Meares était en Chine lorsque Martinez arriva, William Douglas, qui commandait un de ses navires, était toujours dans les parages. Comme le marchand n'avait pas de permis de la East India Company ni de la South Sea Company, qui détenaient conjointement le monopole de la traite dans le Pacifique, ce navire naviguait sous pavillon portugais. Après avoir étudié la traduction des instructions, Martinez jugea qu'elles étaient inacceptables, en particulier parce que, selon lui, Douglas avait l'ordre de résister à tout navire qui tenterait de saisir le sien ou de le détourner de sa route. Cet article «led him to conclude that Spanish claims to sovereignty over the northwest coast were being violated ». (Cook, 1973, p. 151) Martinez arrêta donc Douglas. Le capitaine, évidemment, tenta de persuader le commandant espagnol qu'il se trompait :

I told him, they had not interpreted the Papers right ; that although I did not understand Portuguese, I had seen a Copy of them in English at Macao, which mentioned, if I was attacked by any of those three Nations, to defend myself, and if I had the Superiority, to send the Captain and Crew to Macao to answer for the Insult they offered the Portugueze [sic] Flag. The Padries [sic] and the Clerk read the Papers over, and said they had interpreted the Papers right. (Douglas, s. d., p. 21)

$\mathrm{Au}$ bout de trois semaines, Martinez, qui n'avait pas assez d'hommes pour conduire Douglas et son équipage à San Blas, et qui n'avait pas non plus suffisamment de vivres pour les garder à Nootka, les laissa partir. Il justifia sa décision en disant qu'après mûre réflexion, il avait conclu que les instructions rédigées en portugais pouvaient avoir été mal comprises, car aucun de ceux qui l'accompagnaient ne maîtrisait vraiment cette langue. Mais, comme le fait remarquer Warren Cook, « One suspects Martinez of grasping at Douglas's contention of a linguistic misunderstanding as the easiest way out of his dilemma $»$. (1973, p. 157) En juillet, Martinez, qui avait eu des renforts, saisit deux autres navires de Meares et, cette fois, les prisonniers ne furent pas relâchés.

Tout cela, cependant, avait été fait inutilement, car le commandant espagnol reçut dès le mois d'août l'ordre d'abandonner l'établissement de Nootka. À l'automne, les Amérindiens étaient de 
nouveau seuls; tout était calme sur la côte. En Europe, par contre, c'était loin d'être le cas.

\section{La première convention de Nootka}

Quand Meares apprit en novembre ce qui s'était passé, il partit immédiatement pour l'Angleterre, où l'affaire prit des proportions énormes. En effet, il rédigea un mémoire dans lequel il dénonçait à grands cris les injustices que les Espagnols lui avaient fait subir. Ce mémoire enflamma l'opinion publique, et les membres du Parlement furent même suffisamment impressionnés pour voter les fonds qu'exigerait une guerre contre l'Espagne. L'Espagne envisagea d'abord de riposter, mais quand elle sut qu'elle ne pourrait compter sur la France, alors en pleine révolution, elle fit marche arrière et entama des négociations. La première convention de Nootka fut signée en octobre 1790. L'Espagne s'engageait à rendre les navires saisis, à dédommager Meares et à restituer le territoire que l'Angleterre revendiquait. Toutefois, la convention était imprécise quant à l'étendue de ce territoire, et ce point allait faire l'objet de longues négociations entre George Vancouver et Francisco de la Bodega y Quadra, qui, en 1792, représenteraient leur pays respectif à Nootka pour la restitution officielle.

\section{La seconde occupation de Nootka par les Espagnols}

Au début de 1790, le comte de Revillagigedo, nouveau vice-roi du Mexique, n'était pas encore au courant de ce qui se passait en Europe. En février, il chargea Francisco de Eliza de construire un nouvel établissement à Nootka. Il désirait également qu'on étende les explorations et qu'on les complète par le travail de scientifiques. Les équipages comprenaient des interprètes qui traduisaient vers le russe, d'autres qui traduisaient vers l'anglais et d'autres, enfin, qui connaissaient des langues autochtones. Il semble que le vocabulaire de ces derniers ait parfois été limité : Francisco Xavier de Viana, un des membres de l'expédition d'Alejandro Malaspina, note dans son journal qu'au cours d'une exploration dans les environs de Nootka, des Amérindiens voulaient connaître le but de leur visite, mais qu'ils ne purent leur en donner la moindre idée « porque los intérpretes sólo 
podian merecer este nombre para las cosas familiares ${ }^{11}[\ldots] » .(1958$, p. 92)

Manuel Quimper, quant à lui, explora une grande partie de la côte de l'île de Vancouver. En 1791, alors qu'il faisait voile vers Macao à bord d'un des navires saisis par Martinez pour le rendre à son propriétaire, il était accompagné de l'interprète Juan Kendrick, ainsi que le montre un incident amusant. En effet, rencontrant au large des îles Sandwich James Colnett, capitaine qui avait été fait prisonnier en 1789, il chargea Kendrick de lui porter un message dans lequel il l'assurait de ses bonnes intentions. Mais Colnett, qui se méfiait de l'Espagnol, se prépara quand même à attaquer. Il renvoya la chaloupe, où prenait place un de ses officiers qui apportait une lettre dans laquelle le marchand écrivait que le navire devait lui être remis et qu'il détenait Kendrick jusqu'à ce qu'il sache ce que Quimper avait décidé. Évidemment, comme l'interprète n'était pas là, personne ne put comprendre le message! En désespoir de cause, l'officier britannique, qui parlait un peu espagnol, dit qu'en raison de son rang élevé Colnett insistait pour que toute entrevue ait lieu sur son navire, ce qui n'avait aucun rapport avec le contenu de la lettre, mais n'était pas malgré tout une mauvaise idée : une fois Quimper à bord, les deux capitaines purent de nouveau communiquer par l'intermédiaire de Kendrick, et, finalement, Colnett se contenta de copier les instructions de Quimper. (Colnett, 1940, pp. 213-219; Cook, 1973, p. 299)

\section{Les négociations territoriales}

Au début du printemps 1792, Bodega arrivait à Nootka à la tête d'une expédition dont l'un des membres, le naturaliste José Mariano Moziño, allait rédiger un lexique moachat-espagnol très intéressant ${ }^{12}$.

La copie conservée au Ministerio de Asuntos Exteriores est organisée par sujet, plutôt que selon l'ordre alphabétique. (Moziño, 1970, p. xiii) Le lexique contient 325 entrées, dont une centaine de

\footnotetext{
${ }^{11}$ Traduction libre : «parce que les interprètes ne méritent leur nom que quand il est question de choses familières ».

12 L'Américain Joseph Ingraham avait fait cadeau à Martinez d'un lexique anglais-moachat que celui-ci avait fait traduire par son interprète, Gabriel del Castillo. Manuel Quimper avait lui aussi compilé un lexique bilingue, mais il s'agissait de travaux plus élémentaires. (Moziño, 1970, p. 52, n. 4) 
verbes. Les substantifs sont répartis selon les domaines suivants : la spiritualité, les parties du corps humain, la famille, les infirmités, la maison et son contenu, les animaux, les astres, etc. Les verbes sont regroupés et présentés à la suite des noms. (Ibid., pp. 102-110) Moziño semble être le seul qui ait tenté de comprendre le fonctionnement de la langue des Nootkas. Ainsi, il explique au sujet des verbes :

I have given all of them in the infinitive form although I understand that some are in the present perfect, others in the past, and others, finally, in the future. To the word auco I give the meaning "to eat," when it really means no more than "he eats". With this word I tried to begin to learn the pattern of its conjugation, but the only result of all my diligence was to observe the third person [singular and plural] of the present indicative tense, the three persons of the singular past perfect, and the second person of the imperative. [...] I observed that with some small variations they could be turned into negatives. When an interrogative is formed, [the verb] is combined with the words of the question to form a single expression [...]. (1970, pp. 52-53)

De plus, Moziño avait appris très rapidement le moachat, de sorte qu'il put servir d'interprète à son commandant au cours des nombreuses conversations que celui-ci eut avec Maquinna. (Bodega, en effet, désirait se renseigner, avant l'arrivée de Vancouver, sur tout ce qui s'était passé à Nootka en 1789.) Le naturaliste parlait également portugais et anglais, mais c'est plutôt le capitaine de dragons Gabriel del Castillo qui allait être l'interprète et le traducteur officiel de Bodega pendant les négociations avec Vancouver.

Vancouver arriva à Nootka au mois d'août. Comme il ne parlait pas espagnol, il est étonnant de constater qu'il n'avait pas d'interprète officiel. Il trouva cependant quelqu'un qui lui parut tout à fait compétent en la personne de Thomas Dobson : «On this occasion I considered myself very fortunate in finding a young gentleman (Mr. Dobson) on board the store-ship, who spoke and translated the Spanish language very accurately, and who politely offered me his services. » (Vancouver, 1984, p. 663).

Quelques commentaires de Lamb, éditeur de l'ouvrage de Vancouver, donnent toutefois à penser que les traductions de Dobson n'étaient peut-être pas toujours excellentes. Pour ne citer qu'un exemple, Dobson emploie, dans la traduction d'une lettre de Bodega, l'expression "the above mentioned place» alors que deux noms de lieux sont mentionnés plus haut dans le texte, ce qui induit Vancouver 
en erreur. (v. Vancouver, 1984, p. 665, n. 1). Quoi qu'il en soit, une remarque de Vancouver montre à quel point le traducteur était essentiel: "The letter I received the $2 \mathrm{~d}$ of this month from $\mathrm{Sen}^{\mathrm{r}}$ Quadra, not having been translated till this day [le 10], in consequence of Mr. Dobson's indisposition, I was not a little surprized to find it differ so much from what I had reason to expect. » (Ibid., p. 674)

Aucun renseignement sur la compétence de Gabriel del Castillo n'apparaît dans les sources consultées, mais le fait qu'on avait recours à ses services depuis 1789 permet de supposer qu'il donnait satisfaction.

Malgré leur bonne volonté, Vancouver et Bodega n'arrivèrent pas à s'entendre quant à l'étendue du territoire que l'Espagne devait restituer : il fallut deux autres conventions avant que la question ne soit réglée. Finalement, les deux pays renoncèrent à Nootka, qui devint une zone neutre.

Vancouver et Bodega furent remplacés respectivement par Pearce et par Àlava, qui se rendirent à Nootka en 1795. Àlava ordonna à ses hommes de démanteler le fort et de se préparer à abandonner l'établissement. C'est l'interprète Juan Kendrick qui fut chargé d'expliquer la situation au chef Maquinna. Ainsi que le fait remarquer Warren Cook :

No other person, except possibly Ma-kwee-na, was eyewitness to so much of early northwest coast history. Valuable as the young American should have been as a source of information, not a single document from his hand is known. [...] Sufficiently taciturn to remain unquoted in the sources, Juan Kendrick nonetheless earned the esteem of a succession of Iberian commandants. He was an important link in the bonds of friendship that long endured between Spanish and American nationals on the northwest coast, virtually without a contrary incident. (1973, pp. 428-429)

Howay, qui se fonde sur une lettre de Martinez, dit que cet interprète était « well educated » et « expert as a pilot». (1922, p. 296) Il devait aussi être doué pour les langues puisque dès le mois d'août 1789, soit moins d'un an après son arrivée à Nootka, il servit d'interprète aux Espagnols à l'occasion d'une visite de Maquinna. En 
1796, le jeune homme accompagna un prisonnier anglophone en Espagne. On perd ensuite sa trace ${ }^{13}$.

La troisième convention de Nootka mit fin aux activités des Espagnols au nord de la Californie; les Britanniques continuèrent d'envoyer sur la côte ouest un ou deux navires par année; le nombre de navires américains, quant à lui, ne cessa de croître : il y en eut près de 250 entre 1800 et 1820 . Des marins continueront à servir d'interprètes, mais on engagera aussi des Autochtones en cette qualité. Par exemple, lors du naufrage du Tonquin en 1812, le seul survivant est l'interprète amérindien Kasiascall. C'est lui qui racontera la fin de ce navire à Gabriel Franchère, lequel fera figurer le récit dans son journal de voyage. (1969, pp. 272-274)

\section{Conclusion}

La documentation, qu'il s'agisse des sources anciennes ou des sources contemporaines, ne fournit que des informations fragmentaires - et souvent anecdotiques - sur l'interprétation et la traduction pendant cette période de l'histoire de la Colombie-Britannique, mais il est tout de même possible de tirer quelques conclusions.

L'interprétation ne paraît pas avoir eu une grande importance dans les explorations par voie maritime: les explorateurs devaient reconnaître la côte sur la plus grande distance possible au cours d'un été, et les contacts avec les Amérindiens étaient généralement de courte durée. De plus, contrairement à ceux qui effectueront plus tard des expéditions par voie terrestre ${ }^{14}$, ils n'avaient pas vraiment besoin de communiquer avec les autochtones. En effet, ils ne mettaient pied à terre que pour se réapprovisionner en bois ou en eau, ou encore pour réparer leurs navires: si le capitaine Cook, par exemple, choisit de séjourner quelques semaines à Nootka, c'est avant tout parce que cet endroit offrait un havre bien abrité où il pouvait en toute sécurité radouber ses navires et non parce qu'il était habité.

\footnotetext{
${ }^{13}$ Il y a bien un John Kendrick à bord d'un navire américain ancré à Nootka en 1799 , et un capitaine britannique qui s'y trouve en 1802 porte également ce nom, mais rien n'indique qu'il s'agit de l'interprète. (Howay, 1922, p. 298)

${ }^{14}$ Pour ces explorateurs, l'interprétation fut souvent une véritable question de survie. Voir à ce sujet mon article intitulé «L'interprétation en ColombieBritannique à l'époque des explorations par voie terrestre et de la traite des fourrures dans les comptoirs (1793-1846) », soumis pour publication dans TTR.
} 
Le cas des commerçants est bien différent. Il est clair que la connaissance des langues amérindiennes ou la présence d'une personne qui pouvait servir d'interprète permettait d'obtenir une meilleure récolte de fourrures. Comme les marchands devaient, à l'instar des explorateurs, longer le littoral sur une longue distance, ils ne pouvaient s'arrêter longtemps dans les villages qui les intéressaient. C'est pourquoi bon nombre d'entre eux envisagèrent de laisser certains de leurs hommes parmi les autochtones pendant un été ou même un an, mais ce qui avait réussi à Samuel de Champlain quelque 150 ans plus tôt sur les rives du Saint-Laurent fut un échec sur la côte du Pacifique. Le recours à des Amérindiens comme interprètes ne semble pas, non plus, avoir eu beaucoup de succès. Certains capitaines choisirent alors d'hiverner sur le littoral avec leur équipage, et cette immersion en milieu autochtone paraît avoir donné de bons résultats. Quant aux lexiques bilingues compilés par les marchands, leur nombre même indique qu'on les considérait comme utiles. L'intérêt de ces petits travaux lexicographiques est à souligner. En effet, bien que l'élaboration de listes lexicales bilingues ou multilingues dans un but utilitaire soit une activité fort ancienne ${ }^{15}$, le fait que la plupart des premiers vocabulaires comprenant une des langues amérindiennes parlées sur la côte ouest du Canada aient été élaborés par des marchands pour des raisons commerciales est un phénomène unique en Amérique : partout ailleurs, les premiers lexiques ou dictionnaires mettant en présence une langue autochtone et une autre langue furent compilés par des missionnaires à des fins d'évangélisation ${ }^{16}$.

15 Dès 2000 av. J.-C., il y avait en Mésopotamie de nombreuses tablettes bilingues dans lesquelles figuraient des mots sumériens appartenant à la langue courante et leur équivalent en akkadien. «Ces protorépertoires mettent en correspondance deux langues en vue de répondre aux nécessités pratiques de maintenir la communication entre des collectivités parlant des langues différentes [...] et ayant entre eux [sic] de nombreux échanges économiques, commerciaux, intellectuels, etc. », estime Boulanger. (2003, p. 76)

16 Ainsi, en Nouvelle-France, c'est un récollet qui compila le premier dictionnaire français-huron (Sagard, 1632). En outre, selon Boisson, «le pasteur Roger Williams, fondateur de la colonie du Rhode Island, [publia] à Londres un " phrase book " d'une langue algonquienne maintenant éteinte » dès 1643 et, dans l'Amérique espagnole, les dictionnaires bilingues apparurent à la suite de «décisions ecclésiastiques ». (1996, pp. 24, 28) 
Certains des vocabulaires sont très modestes, et les mots y sont présentés sans aucun ordre particulier. Dans ceux qui sont plus volumineux, c'est un classement thématique qui est privilégié. Parmi les lexiques observés, en effet, un seul est présenté par ordre alphabétique. Quelques auteurs seulement (Cook, Walker, Moziño) accompagnent leur lexique de remarques sur la langue. Par contre, beaucoup mentionnent que l'alphabet romain ne leur permettait pas de rendre adéquatement les sons des langues autochtones. S'ils s'en contentèrent, c'est probablement parce qu'ils n'avaient ni le temps ni la formation qu'aurait exigés la création d'un autre système d'écriture ${ }^{17}$.

Pendant les négociations territoriales, l'interprétation et la traduction ont manifestement été essentielles : toutes les conversations entre Vancouver et Bodega exigeaient la présence d'interprètes, et les nombreuses lettres qu'ils ont échangées devaient elles aussi être traduites. Malheureusement, nous ne savons rien des difficultés de traduction auxquelles durent se heurter Manuel del Castillo, militaire de métier, et Thomas Dobson, marin devenu interprète et traducteur du jour au lendemain.

Pour ce qui est de la qualité de l'interprétation et de la traduction à cette époque, enfin, certaines remarques permettent de croire qu'elle laissait parfois à désirer. Néanmoins, à une ou deux exceptions près, on semble avoir apprécié les services de ceux qui jouaient le rôle d'interprètes ou de traducteurs. Cela ne surprend pas quand on constate ce qui se passait en leur absence : ainsi, en 1793, un des navires de Vancouver avait besoin d'être radoubé et les charpentiers espagnols prêtèrent main-forte aux charpentiers britanniques, mais l'interprète ne pouvait être partout à la fois,

[...] and "when this Man is not at hand," Manby remarks, "curious scenes, repeatedly ensue as our Conversation is generally carried on, by a few words, of all Languages $-\&$ signs [...] some times, we understand each other-at other times not: The honest Don [Fidalgo], slaps his forehead, shrugs his Shoulders and exclaims :

\footnotetext{
${ }^{17}$ Ce sont des missionnaires qui inventeront, beaucoup plus tard, des écritures adaptées aux langues parlées à l'ouest des Rocheuses canadiennes. Voir à ce sujet mon article intitulé "Colombie-Britannique: les missionnaires catholiques et les activités langagières (1842-1952)», accepté pour publication dans le numéro spécial de Meta «Histoire de la traduction et traduction de l'histoire ».
} 
Diable What a pity; what a misfortune... that so many Languages should prevail in this little World." (Cook, 1973, p. 398)

Université Laval 


\section{ANNEXE}

Colombie-Britannique : les nations du littoral et les langues ou dialectes parlés à l'époque observée

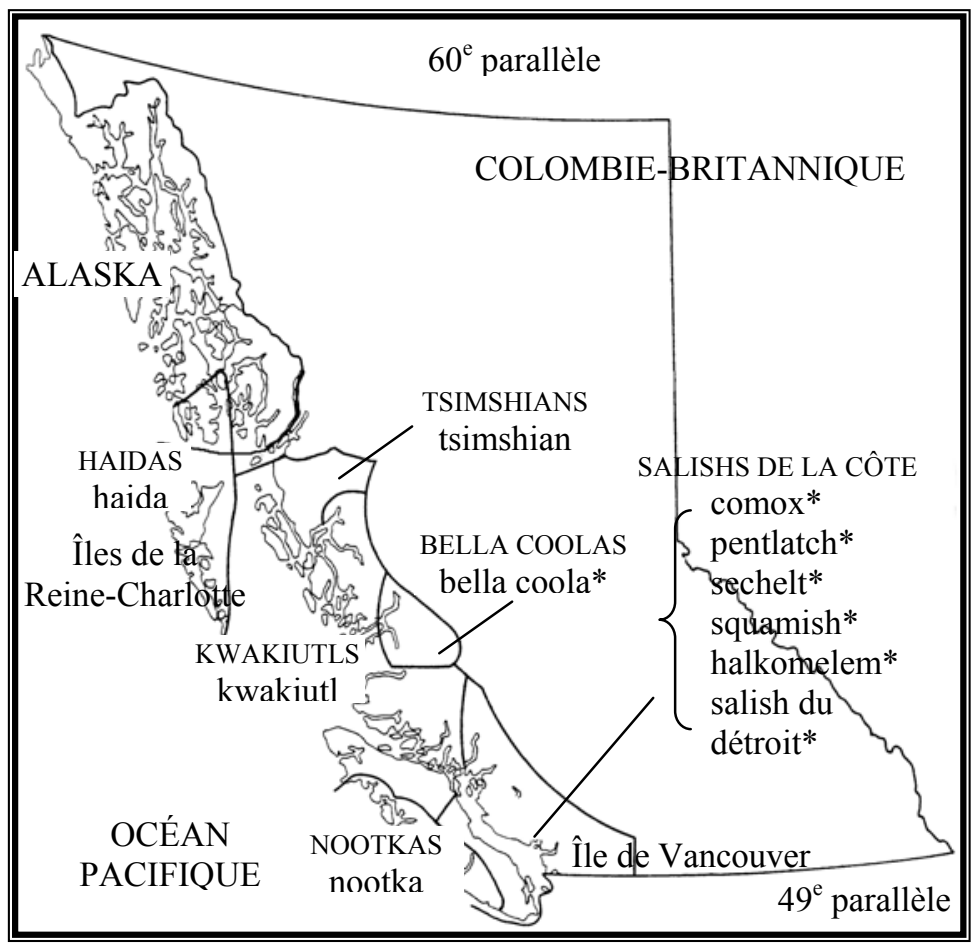

* dialecte appartenant à la famille salishenne

Sources des renseignements ethnographiques et linguistiques: Affaires indiennes et du Nord Canada, 1997, pp. 37-39; Duff, 1969, pp. 12-15; Woodcock, 1990, pp. 3-4. 


\section{Références}

$\underline{\text { Sources primaires }}$

BODEGA Y QUADRA, Juan Francisco (1943). « Navegación hecha por don Juan Francisco de la Bodega y Quadra, Teniente de Fregata de la Real Armada y Comandante de la Goleta "Sonora"; a los descubrimientos de los Mares y Costa Septentrional de California. Colección de diarios y relationes para la historia de los viajes y descubrimientos. Madrid, Instituto histórico de marina, pp. 97-133.

[COLNETT, James] (1940). The Journal of Captain James Colnett Aboard the Argonaut from April 26, 1789 to Nov. 3, 1791. Édité par F. W. Howay. Toronto, The Champlain Society.

COOK, James (1821). The Three Voyages of Captain James Cook Round the World. London, Printed for Longman, Hurst, Rees, Orme, and Brown.

- (1967). The Journals of Captain James Cook. Vol. 3 The Voyage of the Resolution and Discovery. 1776-1780. Édité par J. C. Beaglehole. Cambridge, The Hakluyt Society.

[CRESPI, Fray Juan] (1969). « Journal of Fray Juan Crespi kept during the same voyage [voyage de Juan Pérez]-dated $5^{\text {th }}$ October, $1774 »$. The California Coast. A Bilingual Edition of Documents From the Sutro Collection. Traduit et édité par Donald C. Cutter. Norman, University of Oklahoma Press, pp. 202-278.

DOUGLAS, William (s. d.). " Extract of the Journal of the Iphigenia ». Mr. Mears's [sic] Memorial. Dated 30th April, 1790. s. 1., s. é.

[FRANCHÈRE, Gabriel] (1969). The Journal of Gabriel Franchère. Édité par W. Kaye Lamb. Toronto, The Champlain Society.

[HASWELL, Robert] (1941). "Log of the First Voyage of the Columbia ». Voyages of the "Columbia" to the Northwest Coast 17871790 and 1790-1793. Édité par F. W. Howay. Boston, The Massachusetts Historical Society, pp. 2-107.

[INGRAHAM, Joseph] (1971). Joseph Ingraham's Journal of the Brigantine Hope on a Voyage to the Northwest Coast of North 
America. Édité par Mark D. Kaplanoff. Barre, Massachusetts, The Imprint Society.

MEARES, John (1967). Voyages Made in the Years 1788 and 1789 from China to the North-West Coast of America. Amsterdam-NewYork, N. Israel-Da Capo Press.

MOURELLE, Francisco Antonio (1781). Journal of a Voyage in 1775 to Explore the Coast of America, Northward of California by the Second Pilot of the Fleet, Don Francisco Antonio Maurelle [i.e. Mourelle], in the King's Schooner Called the Sonora, and Commanded by Don Juan Francisco de la Bodega. [Londres], s. é.

MOZIÑO, José Mariano (1970). Noticias de Nutka: An account of Nootka Sound in 1792. Traduit et édité par Iris Higbie Wilson. Seattle et Londres, University of Washington Press.

[PEÑA, Fray Tomás de la], (1969). « Diary of Fray Tomás de la Peña kept during the voyage of the Santiago-dated $28^{\text {th }}$ August, $1774 »$. The California Coast. A Bilingual Edition of Documents From the Sutro Collection. Traduit et édité par Donald C. Cutter. Norman, University of Oklahoma Press, pp. 135-201.

SAGARD, Gabriel (1632). Dictionaire [sic] de la langue huronne, Nécessaire à ceux qui n'ont l'intelligence d'icelle \& ont à traiter avec les sauvages du pays. Paris, Denys Moreau.

[STRANGE, James] (1982). James Strange's Journal And Narrative of the Commercial Expedition from Bombay to the Northwest Coast of America. Fairfield, Ye Galleon Press.

[STURGIS, William] (1978). The Journal of William Sturgis. Édité par S. W. Jackman. Victoria, Sono Nis Press.

- (2000). "The Character of Northwest Indians and Occurrences Among them ". "A Most Remarkable Enterprise". Lectures on the Northwest Coast Trade and Northwest Coast Indian Life by Captain William Sturgis. Édité par Mary Malloy. Marston Mills, Massachusetts, Parnassus Imprints, pp. 25-50. 
VANCOUVER, George (1984). A Voyage of Discovery to the North Pacific Ocean and Round the World 1791-1795. Édité par W. Kaye Lamb. Londres, The Hakluyt Society.

VIANA, Francisco Xavier de (1958). Diario de viaje. Montevideo, Ministerio de Instrucción Pública y Previsión Social.

WALKER, Alexander (1982). An Account of a Voyage to the North West Coast of America in 1785 \& 1786. Édité par Robin Fisher et J. M. Bumsted. Vancouver-Toronto, Douglas \& McIntyre; Seattle, University of Washington Press.

\section{$\underline{\text { Sources secondaires }}$}

Affaires indiennes et du Nord Canada (1997). Les Premières nations du Canada. Ottawa, Affaires indiennes et du Nord Canada.

ARCHER, Christon E. (1993). "Seduction before Sovereignty: Spanish Efforts to Manipulate the Natives in Their Claims to the Northwest Coast ». From Maps to Metaphors. The Pacific World of George Vancouver. Robin Fisher et Hugh Johnston (dir.). Vancouver, UBC Press, pp. 127-159.

BARMAN, Jean (1996). The West Beyond the West. A History of British Columbia. Toronto-Buffalo-Londres, University of Toronto Press.

BOISSON, Claude (1996). «L'antiquité et la variété des dictionnaires bilingues ». Les dictionnaires bilingues. Louvain-la-Neuve, AupelfUref - Éditions Duculot.

BOULANGER, Jean-Claude (2003). Les inventeurs de dictionnaires. De l'eduba des scribes mésopotamiens au scriptorium des moines médiévaux. Ottawa, Les Presses de l'Université d'Ottawa.

COOK, Warren L. (1973). Flood Tide of Empire. Spain and the Pacific Northwest, 1543-1819. New Haven-Londres, Yale University Press.

DONALD, Leland (1997). Aboriginal Slavery on the Northwest Coast of North America. Berkeley-Los Angeles, University of California Press. 
DUFF, Wilson (1969). The Indian History of British Columbia. Victoria, Royal British Columbia Museum.

FISHER, Robin (1996). «Contact and Trade, 1774-1849». The Pacific Province. A History of British Columbia. Hugh J. M. Johnston (dir.). Vancouver-Toronto, Douglas \& McIntyre, pp. 48-67.

GIBSON, James R. (1992). Otter Skins, Boston Ships and China Goods. The Maritime Fur Trade of the Northwest Coast, 1785-1841. Montréal-Kingston-Londres, Mcgill-Queen's University Press.

HOWAY, Frederic W. (1922). «John Kendrick and His Sons». Oregon Historical Society Quarterly, 23, 4, pp. 277-302.

MARSHALL, Yvonne (1993). «Dangerous Liaisons: Maquinna, Quadra and Vancouver in Nootka Sound, 1790-5 ». From Maps to Metaphors. The Pacific World of George Vancouver. Robin Fisher et Hugh Johnston (dir.). Vancouver, UBC Press, pp. 160-175.

WOODCOCK, George (1994). British Columbia. A History of the Province. Vancouver-Toronto, Douglas \& McIntyre.

\section{Bibliographie}

Sources primaires

BARTLETT, John (1925). «A Narrative of Events in the Life of John Bartlett of Boston, Massachusetts, in the Years 1790-1793, During Voyages to Canton and the Northwest Coast of America ». The Sea, the Ship and the Sailor. Tales of Adventure from Log Books and Original Narratives. Salem, Massachusetts, Marine Research Society, pp. 287337.

[BISHOP, Charles] (1967). The Journals and Letters of Captain Charles Bishop on the North-West Coast of America, in the Pacific and in New South Wales 1794-1799. Édité par Michael Roe. Cambridge, Cambridge University Press.

[BOIT, John] (1981). Log of the Union. John Boit's Remarkable Voyage to the Northwest Coast and Around the World 1794-1796. Portland, Oregon Historical Society. 
DIXON, George (1968). A Voyage Round the World: But More Particularly to the North-west Coast of North America. Amsterdam New-York, N. Israel - Da Capo Press.

[JOHNSON, Ebenezer] (1974). A Short Account of a Northwest Voyage performed in the years 1796, 1797,\& 1798 by Ebenezer Johnson. Édité par M. S. Batts. Vancouver, The Alcuin Society.

[STURGIS, William] (2000). " An Historical Sketch of the Northwest Coast Trade". "A Most Remarkable Enterprise". Lectures on the Northwest Coast Trade and Northwest Coast Indian Life by Captain William Sturgis. Édité par Mary Malloy. Marston Mills, Massachusetts, Parnassus Imprints, pp. 1-21.

$\underline{\text { Sources secondaires }}$

EFRAT, Barbara S. (1978). "Linguistic Acculturation on the West Coast of Vancouver Island ». Sound Heritage, 7, 1, pp. 89-98.

FISHER, Robin (1992). Contact and Conflict. Vancouver, UBC Press.

HOWAY, Frederic W. (1923). « Early Days of the Maritime Fur-Trade on the Northwest Coast ». The Canadian Historical Review, 4, pp. 2644.

McKELVIE, B. A. (1946). Maquinna the Magnificent. Vancouver, The Vancouver Daily Province.

PETHICK, Derek (1976). First Approaches to the North-West Coast. Vancouver, J. J. Douglas Ltd.

RUBY, Robert H. et John A. BROWN (1993). Indian Slavery in the Pacific Nortwest. Spokane, The Arthur H. Clark Company.

SCHWANTES, Carlos Arnaldo (1996). The Pacific Northwest. Lincoln et Londres, University of Nebraska Press.

RÉSUMÉ : L'interprétation et la traduction sur la côte ouest du Canada actuel à l'époque des explorations par mer et des débuts de la traite des fourrures sur le littoral (1774-1800) — L'interprétation n'a pas joué un grand rôle au cours des explorations par voie maritime, 
les contacts avec les Amérindiens étant la plupart du temps très brefs. Le cas des marchands est différent. Comme la récolte de fourrures était plus abondante quand on pouvait communiquer avec les autochtones autrement que par signes, l'interprétation a vite pris de l'importance. En outre, presque tous les marchands ont laissé au moins un lexique bilingue. Au cours de la crise de Nootka, au début des années 1790, toutes les négociations territoriales menées par Vancouver, qui représentait la Grande-Bretagne, et Bodega, qui représentait l'Espagne, ont dû être traduites. L'interprétation et la traduction y ont donc joué un rôle essentiel.

\begin{abstract}
Interpreting and Translation on the Canadian West Coast at the Time of the Maritime Explorations and the Early Days of the Fur Trade on the Coast, 1774-1800 - Interpreting did not play a significant part in the explorations by sea because contacts with the natives were brief. Fur traders, though, realised that they could obtain more furs when they could communicate with the natives through interpreters. Many merchants also tried to learn native languages and compiled bilingual vocabularies. During the Nootka controversy, in the early 1790s, talks and letters between Vancouver and Bodega had to be translated: therefore, interpreting and translation were absolutely necessary.
\end{abstract}

Mots-clés : interprétation, traduction, lexicographie, ColombieBritannique, XVIII ${ }^{\mathrm{e}}$ siècle.

Keywords: interpreting, translation, lexicography, British Columbia, $18^{\text {th }}$ century.

Ginette Demers : Département de langues, linguistique et traduction, Université Laval, Québec (Québec) G1K 7P4

Courriel : Ginette.Demers@lli.ulaval.ca 